tween these poles. This is what Florida Atlantic University's system aims at; if it achieves this as well as the synthesis between the advantages and disadvan- tages of the computer, the efforts at University of Illinois, Chicago and Florida Atlantic University will be vindicated.

\title{
Cataloging for Document Retrieval at Florida Atlantic University
}

\author{
BY CLAYTON D. HIGHUM
}

INCLUDED in the cataloging of library materials at Florida Atlantic University is a procedure which involves the coding of specific information for the purpose of later retrieval of these materials. This coding is done on the same transmittal sheet that is used for the descriptive cataloging of materials for the computer-produced book catalog. As is indicated in the form reproduced here, three fortyspace columns are utilized for the coding of the desired information. The entire procedure may be described quite accurately as an expansion of the collation and imprint of a document. The reason for the forty-space columns instead of the eighty-space columns of the typical IBM data processing card is that the length of each column was determined somewhat by the availability of space on the transmittal sheet. Also, future expansion of the procedure will be possible without a complete reformatting of the transmittal sheet.

Some explanation for the use of the term "document" in this article might be in order. The reader will realize that the coding areas on the transmittal form include many elements not ordinarily associated with a document. First, for lack of a better term, "document" is used. Second, the retrieval discussed in this article includes types of materials other than the typical document. The library at Florida Atlantic University is an integral part of the Learning Resources Di-
Mr. Highum is Assistant Librarian in Cataloging at Florida Atlantic University.

vision, and as such the catalog will include all materials, book and nonbook, that comprise the collection of the entire division. Therefore, rather than attempting continually to differentiate among the many forms of material included on the coding sheet, the word "document" is being used as an all-inclusive term.

Most of the fields represented on the coding sheet are self-explanatory. Therefore, no detailed explanation will be given here. Detailed instructions have been developed, however, and are given to the catalogers to assist them in the coding.

In general terms, each labeled space that can be associated with the document being cataloged is either checked or filled with an appropriate, pre-established code. The only limit to the number of spaces checked is the document being cataloged. It is assumed that the catalogers will analyze each piece of material carefully enough to insure adequate coverage. Obviously, the more specific the analysis, the more meaningful and worthwhile a retrieval becomes to the patron. Some blank spaces have been left on the form to allow for future expansion or for the addition of elements that were forgotten or not justified for inclusion at the time the form was developed.

Field 1-5 in area 81 provides space for the indication of the type of main entry 


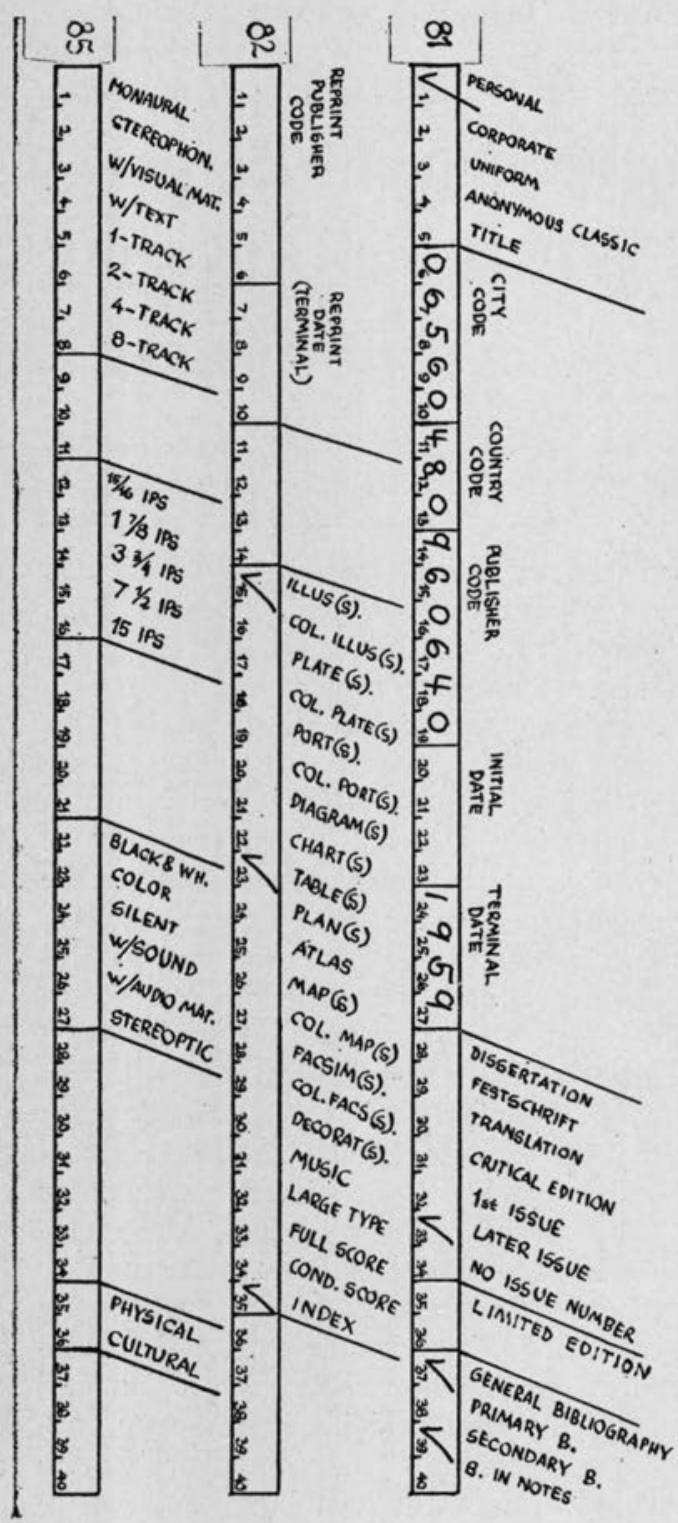

The above coding was done for the following source and is representative of the average amount done on a book of this type.

Lamanna, Carl.

Basic bacteriology, its biological and chemical background [by] Carl Lamanna [and] M. Frank Mallette. 2d ed. Baltimore, Williams and Wilkins [1959] associated with a document. A check mark is placed in the appropriate space.

Field 6-27 represents the imprint of a document in coded form. These codes have been developed and are in the form of alphabetical lists by city, publisher, and country. The cataloger refers to these lists for the appropriate information. Each entry in the list is given an identification number which becomes the code entered in the spaces provided on the transmittal sheet. Data to be inserted in this field are for the original source of the document. If the document is a reprint, the data are inserted in area 82 , field 1-10.

The dates to be entered in $81: 20-27$ are those for the original issue of the document or documents being cataloged. For example, if a set of books includes volumes that were published at various times, the date of the first volume is placed in "Initial Date" (20-23), and the date for the most recent volume is placed in spaces 24-27. The "Terminal Date" of the set will be revised as more recent volumes are purchased. Single works or sets issued in a single year will be indicated with a terminal date only.

Field 28-34 of area 81 is reserved for edition data. The word "issue" is used here to eliminate the confusion that sometimes results from an attempt to define the term "edition" to the satisfaction of all concerned.

Field $37-40$ is provided to indicate the inclusion of bibliographic data and to identify, more specifically, the types of bibliography in a document. This field is an example of one in which a number of spaces could be checked for a single document.

In area 82 , field $1-10$ provides space to code data pertaining to a reprint. A reprint is defined, for coding purposes, as being a re-issue of a work from the original plates by some publisher other than the first.

A section is provided by field $15-35$ in 
which the type of illustrative material in a document may be designated. All spaces which apply to the document are checked rather than the more general types of illustration eliminated in favor of more specific. Therefore, if a book is fully illustrated, a cataloger could expect to check ten to twelve spaces in this field to describe adequately the illustrations in the document and to insure as being more useful any retrieval requested, either partially or completely, on the basis of the illustrative material. Distinctions among various types of illustrations are made according to the definitions in the cataloging instruction manual.

Spaces are provided in area 85 for the coding of descriptive data that pertains primarily to nonbook material. One will notice, however, that spaces 26,35 , and 36 will often be used when coding printed material. Here, again, the instructions for the coding and the definitions for each heading are found in the instruction manual.

The information from the transmittal sheet is key-punched into data processing cards and ultimately read onto magnetic tape as a part of the descriptive cataloging information for a specific document. The cataloged data is stored on the magnetic tape according to the $\mathrm{Li}$ brary of Congress classification of the document, thus making possible a search of each cataloged item in the collection for specific information.

The practical result of the coding for document retrieval will be the production of bibliographies and lists of library materials for students, faculty members, and librarians that will conform to their speci- fications in so far as the type of information they desire has been coded. One must remember that this coding procedure is not an attempt at information retrieval but an attempt at efficient and more worthwhile document retrieval.

A faculty member might ask for all first editions of an author, published in Germany during a specified period of time, with primary bibliography, and expect to receive a printed list of materials in the library collection which meet his requirements.

A student who is interested in illustrations might ask for the materials of certain organizations known to be involved in the publishing of well illustrated books, limit the request by date of publication and specify colored portraits, colored plates, and colored maps. He also will receive a list of library materials that meet his requirements.

One finds it possible to believe that the librarians at Florida Atlantic University may find much practical value in being able to cull from the collection, quickly and efficiently, special lists of materials that could greatly expedite the checking of certain specialized proposed purchases against the existing collection, or of special lists that might become excellent tools for the reference department.

The patrons of the Florida Atlantic University library who wish to avail themselves of the retrieval service will be provided with the necessary guidance to understand what types of information have been coded and the definitions of the coded headings. Obviously, such information will be essential if any worthwhile results are expected from a retrieval. 